\title{
Conformal Vector Fields on Some Finsler Manifolds
}

\author{
Zhongmin Shen and Mingao Yuan
}

July 8, 2015

\begin{abstract}
In this paper, we study conformal vector fields on a Finsler manifold whose metric is defined by a Riemannian metric, a 1-form and its norm. We find PDEs characterizing conformal vector fields. Then we obtain the explicit expressions of conformal vector fields for certain spherically symmetric metrics on $R^{n}$.

Key words: General $(\alpha, \beta)$-metrics, Conformal vector field, Spherically symmetric metrics
\end{abstract}

Mathematics Subject Classification 2000: 53B40, 53C60

\section{Introduction}

Conformal vector fields are important in Riemann geometry and RiemannFinsler geometry. We can use them to solve other problems in Riemann-Finsler geometry. Let $(M, F)$ be a Finsler manifold. It is known that a vector field $V=V^{i} \frac{\partial}{\partial x^{i}}$ on $M$ is a conformal vector field of $F$ with conformal factor $c=c(x)$ if and only if

$$
X_{V}\left(F^{2}\right)=4 c F^{2}
$$

where $X_{V}:=V^{i} \frac{\partial}{\partial x^{i}}+y^{i} \frac{\partial V^{j}}{\partial x^{i}} \frac{\partial}{\partial y^{j}}(\underline{[7]})$.

Recently, the first author and Q. Xia have studied conformal vector fields on Randers manifold with certain curvature properties. They determine all conformal vectors fields on Randers manifold of weakly isotropic flag curvature ([7]). They also determine conformal vector fields on locally projectively flat Randers manifold $([9])$. Besides, they use homothetic vector fields $(c=$ constant $)$ on Randers manifolds to construct new Randers metrics of scalar flag curvature in ([8]).

In this paper, we shall study conformal vector fields on a manifold $M$ with a general $(\alpha, \beta)$-metric. The so-called general $(\alpha, \beta)$ - metrics are defined in the following form

$$
F=\alpha \phi\left(b^{2}, \frac{\beta}{\alpha}\right)
$$

where $\alpha=\sqrt{a_{i j} y^{i} y^{j}}$ is a Riemann metric, $\beta=b_{i} y^{i}$ is a 1 -form and $b:=\left\|\beta_{x}\right\|_{\alpha}$ is its norm. This kind of metrics are first discussed by C. Yu and H. Zhu ([11]). 
Many well-known Finsler metrics are general $(\alpha, \beta)$-metrics. For example, the Randers metrics and the square metrics are defined by functions $\phi=\phi\left(b^{2}, s\right)$ in the following form:

$$
\begin{aligned}
\phi & =\frac{\sqrt{1-b^{2}+s^{2}}+s}{1-b^{2}} \\
\phi & =\frac{\left(\sqrt{1-b^{2}+s^{2}}+s\right)^{2}}{\left(1-b^{2}\right)^{2} \sqrt{1-b^{2}+s^{2}}}
\end{aligned}
$$

In this paper, we shall prove the following:

Theorem 1.1 Let $F=\alpha \phi\left(b^{2}, s\right), s=\beta / \alpha$, be a general $(\alpha, \beta)$-metric on an $n$-dimensional manifold $M(n \geq 3)$. Let $\phi=a_{0}+a_{1} s+a_{2} s^{2}+o\left(s^{2}\right)$, where $a_{i}=a_{i}\left(b^{2}\right)$. Suppose $a_{1} \neq 0$ and

$$
\frac{1}{2 b^{2}}+\frac{a_{1}^{\prime}}{a_{1}}-\frac{a_{0}^{\prime}}{a_{0}}+\left\{\frac{a_{2}}{a_{0}}\left[2 \frac{a_{1}^{\prime}}{a_{1}}-\frac{a_{0}^{\prime}}{a_{0}}\right]-\frac{a_{2}^{\prime}}{a_{0}}\right\} b^{2} \neq 0,
$$

where $a_{i}^{\prime}=\frac{d}{d b^{2}}\left(a_{i}\right)$, then $V=V^{i} \frac{\partial}{\partial x^{i}}$ is a conformal vector field of $F$ with conformal factor $c=c(x)$ if and only if $X_{V}\left(b^{2}\right)=0$ and

$$
V_{i ; j}+V_{j ; i}=4 c a_{i j}, \quad V^{j} b_{i ; j}+b^{j} V_{j ; i}=2 c b_{i} .
$$

Here, $X_{V}\left(b^{2}\right):=V^{i} \frac{\partial b^{2}}{\partial x^{i}}, V_{i}=a_{i k} V^{k}, b^{i}=a^{i k} b_{k}$ and ";" denotes horizontal covariant derivative with respect to $\alpha$.

Note that if $\phi=\phi(s)$ is independent of $b^{2}$, i.,e. $F=\alpha \phi(s), s=\beta / \alpha$ is an $(\alpha ., \beta)$-metric, then (3) always holds since $a_{i}^{\prime}=0$. Thus Theorem 1.1 is a generalization of a result in [3] for $(\alpha, \beta)$-metrics. The functions $\phi=\phi\left(b^{2}, s\right)$ in (1) and (2) both satisfy the same equation:

$$
\frac{1}{2 b^{2}}+\frac{a_{1}^{\prime}}{a_{1}}-\frac{a_{0}^{\prime}}{a_{0}}+\left\{\frac{a_{2}}{a_{0}}\left[2 \frac{a_{1}^{\prime}}{a_{1}}-\frac{a_{0}^{\prime}}{a_{0}}\right]-\frac{a_{2}^{\prime}}{a_{0}}\right\} b^{2}=\frac{1}{2 b^{2}\left(1-b^{2}\right)} \neq 0 .
$$

Theorem 1.1 can be also viewed as an extension of Proposition 3.1 in [7] where it is proved for a special function $\phi$ in (1).

There are functions $\phi=\phi\left(b^{2}, s\right)$ which do not satisfy (3). For such functions, $X_{V}\left(b^{2}\right) \neq 0$ for conformal vector fields $V$. In fact, we can show that

Theorem 1.2 Let $F=\alpha \phi\left(b^{2}, s\right), s=\beta / \alpha$, be a general $(\alpha, \beta)$-metric on an $n$-dimensional manifold $M(n \geq 3)$. Let $\phi=a_{0}+a_{1} s+a_{2} s^{2}+o\left(s^{2}\right)$, where $a_{i}=a_{i}\left(b^{2}\right)$. Suppose $a_{1} \neq 0$ and $\phi=\phi\left(b^{2}, s\right)$ satisfies .

$$
\frac{1}{2 b^{2}}+\frac{a_{1}^{\prime}}{a_{1}}-\frac{a_{0}^{\prime}}{a_{0}}+\left\{\frac{a_{2}}{a_{0}}\left[2 \frac{a_{1}^{\prime}}{a_{1}}-\frac{a_{0}^{\prime}}{a_{0}}\right]-\frac{a_{2}^{\prime}}{a_{0}}\right\} b^{2}=0 .
$$

Then $V=V^{i} \frac{\partial}{\partial x^{i}}$ is a conformal vector field of $F$ with conformal factor $c=c(x)$ if and only if

$$
\begin{aligned}
V_{i ; j}+V_{j ; i} & =4 \bar{c} a_{i j}-2 X_{V}\left(b^{2}\right) b^{-2}\left[\frac{1}{2 b^{2}}+\frac{a_{1}^{\prime}}{a_{1}}-\frac{a_{0}^{\prime}}{a_{0}}\right] b_{i} b_{j}, \\
V^{j} b_{i ; j}+b^{j} V_{j ; i} & =2 \tilde{c} b_{i} .
\end{aligned}
$$


where $\bar{c}:=c-\frac{1}{2} X_{V}\left(b^{2}\right) \frac{a_{0}^{\prime}}{a_{0}}$ and $\tilde{c}:=c-\frac{1}{2} X_{V}\left(b^{2}\right) \frac{a_{1}^{\prime}}{a_{1}}$.

There is a special class of general $(\alpha, \beta)$-metrics called spherically symmetric metrics, which are defined on an open subset of $R^{n}$ with $\alpha=|y|$ and $\beta=\langle x, y\rangle$,

$$
F=|y| \phi\left(|x|^{2}, \frac{\langle x, y\rangle}{|y|}\right) .
$$

Spherically symmetric metrics are studied by many people. Some new examples of Finsler metrics with special curvature properties are constructed in terms of spherically symmetric metrics([2][5]).

Spherically symmetric metrics are a little special, so that one can easily determine conformal vector fields when $\phi=\phi\left(b^{2}, s\right)$ satisfies (3). We get the following result.

Theorem 1.3 Let $F=|y| \phi\left(|x|^{2},\langle x, y\rangle /|y|\right)$ be a spherically symmetric metric on $R^{n}$. Let $\phi=a_{0}\left(|x|^{2}\right)+a_{1}\left(|x|^{2}\right) s+a_{2}\left(|x|^{2}\right) s^{2}+o\left(s^{2}\right)$ with $a_{1} \neq 0$. Suppose that

$$
\frac{1}{2|x|^{2}}+\frac{a_{1}^{\prime}}{a_{1}}-\frac{a_{0}^{\prime}}{a_{0}}+\left\{\frac{a_{2}}{a_{0}}\left[2 \frac{a_{1}^{\prime}}{a_{1}}-\frac{a_{0}^{\prime}}{a_{0}}\right]-\frac{a_{2}^{\prime}}{a_{0}}\right\}|x|^{2} \neq 0 .
$$

then $V$ is a conformal vector field of $F$ if and only if

$$
V^{i}=q_{k}^{i} x^{k}
$$

where $\left(q_{k}^{i}\right)$ is an antisymmetric constant matrix. In this case, the conformal factor $c=0$.

However, when $\phi=\phi\left(b^{2}, s\right)$ satisfies (5), it is difficult to solve (6) and (7) for conformal vector fields. Thus we shall consider more special functions $\phi=$ $a_{0}+a_{1} s+a_{2} s^{2}+o\left(s^{2}\right)$ whose coefficients are given by

$$
a_{0}:=k_{0}|x|^{2 \delta-1}, \quad a_{1}=k_{1}|x|^{2 \delta-2}, \quad a_{2}=k_{2}|x|^{2 \delta-3},
$$

where $k_{0}>0, k_{1} \neq 0, k_{2}$ and $\delta \neq 0$ are constants. Then $a_{0}, a_{1}$ and $a_{2}$ satisfy

$$
\frac{1}{2|x|^{2}}+\frac{a_{1}^{\prime}}{a_{1}}-\frac{a_{0}^{\prime}}{a_{0}}+\left\{\frac{a_{2}}{a_{0}}\left[2 \frac{a_{1}^{\prime}}{a_{1}}-\frac{a_{0}^{\prime}}{a_{0}}\right]-\frac{a_{2}^{\prime}}{a_{0}}\right\}|x|^{2}=0 .
$$

We have the following

Theorem 1.4 Let $F=|y| \phi\left(|x|^{2}, \frac{\langle x, y\rangle}{|y|}\right)$ be a spherically symmetric metric on $R^{n}$. Let $\phi=a_{0}\left(|x|^{2}\right)+a_{1}\left(|x|^{2}\right) s+a_{2}\left(|x|^{2}\right) s^{2}+o\left(s^{2}\right)$ where $a_{0}, a_{1}$ and $a_{2}$ are given by (10). Then $V$ is a conformal vector field of $F$ with conformal factor $c$ if and only if $c=$ constant and

$$
V^{i}=\frac{c}{\delta} x^{i}+q_{k}^{i} x^{k},
$$

where $\left(q_{k}^{i}\right)$ is an antisymmetric constant matrix. 


\section{Preliminaries}

In this paper, we consider a special class of Finsler metrics on a manifold, which are defined by a Riemannian metric $\alpha=\sqrt{a_{i j}(x) y^{i} y^{j}}$, a 1 -form $\beta=b_{i}(x) y^{i}$ and its norm $b:=\sqrt{a^{i j}(x) b_{i}(x) b_{j}(x)}$. They can expressed by

$$
F=\alpha \phi\left(b^{2}, \frac{\beta}{\alpha}\right)
$$

where $\phi=\phi(r, s)$ is a positive smooth functions on $\left[0, b_{o}\right) \times\left(-b_{o}, b_{o}\right)$. It is required that

$$
\phi-\phi_{2} s>0, \quad \phi-\phi_{2} s+\left(b^{2}-s^{2}\right) \phi_{22}>0,
$$

for $|s| \leq b<b_{0}$, where $\phi_{1}:=\frac{\partial \phi}{\partial b^{2}}, \phi_{2}:=\frac{\partial \phi}{\partial s}, \phi_{22}:=\frac{\partial^{2} \phi}{\partial s^{2}}$. Under the above condition, $F$ is a positive definite Finsler metric. Such a Finsler metric is called general $(\alpha, \beta)$ - metrics ([11]).

This class of Finsler metrics is very rich. Many Finsler metrics of constant flag curvature are spherically symmetric, in particular, they are general $(\alpha, \beta)$ metrics $([2],[4], \underline{[5]}, \underline{[6]}$ and $\underline{[12]})$.

Write the function $\phi=\phi(r, s)$ in the following Taylor expansion

$$
\phi=a_{0}+a_{1} s+a_{2} s^{2}+o(s),
$$

where $a_{i}=a_{i}\left(b^{2}\right)$. (13) implies that

$$
a_{0}>0, \quad a_{0}+2 b^{2} a_{2}>0 .
$$

But no restriction on $a_{1}$. If we assume that $a_{1} \neq 0$, then $F$ is not reversible. Consider a Randers metric $F=\alpha+\beta$. The function $\phi=1+s$. We have $a_{0}=1$, $a_{1}=1$ and $a_{i}=0$ for all $i>1$. The Randers metric $F$ can also be expressed as a general $(\alpha, \beta)$-metric in the form (1).

$$
\phi=\frac{\sqrt{1-b^{2}+s^{2}}+s}{1-b^{2}} .
$$

We have

$$
a_{0}=\frac{1}{\left(1-b^{2}\right)^{1 / 2}}, \quad a_{1}=\frac{1}{1-b^{2}}, \quad a_{2}=\frac{1}{2\left(1-b^{2}\right)^{3 / 2}} .
$$

Then we get

$$
\frac{1}{2 b^{2}}+\frac{a_{1}^{\prime}}{a_{1}}-\frac{a_{0}^{\prime}}{a_{0}}+\left\{\frac{a_{2}}{a_{0}}\left[2 \frac{a_{1}^{\prime}}{a_{1}}-\frac{a_{0}^{\prime}}{a_{0}}\right]-\frac{a_{2}^{\prime}}{a_{0}}\right\} b^{2}=\frac{1}{2 b^{2}\left(1-b^{2}\right)} .
$$

It is surprised to see that the function $\phi$ in (2) satisfies (14) too !

\section{Proofs of Theorems 1.1 and $\mathbf{1 . 2}$}

Let $V$ be a conformal vector field of $F$ with conformal factor $c(x)$, that is

$$
X_{V}\left(F^{2}\right)=4 c F^{2} .
$$


For $F=\alpha \phi\left(b^{2}, \beta / \alpha\right)$, we have

$$
\begin{aligned}
X_{V}\left(F^{2}\right) & =X_{V}\left(\alpha^{2} \phi^{2}\right) \\
& =\phi^{2} X_{V}\left(\alpha^{2}\right)+\alpha^{2} X_{V}\left(\phi^{2}\right) \\
& =\phi^{2} X_{V}\left(\alpha^{2}\right)+2 \phi \alpha^{2} \phi_{1} X_{V}\left(b^{2}\right)+2 \phi \phi_{2} \alpha X_{V}(\beta)-2 \phi \phi_{2} \beta X_{V}(\alpha) \\
& =\phi\left(\phi-\phi_{2} s\right) X_{V}\left(\alpha^{2}\right)+2 \alpha \phi \phi_{2} X_{V}(\beta)+2 \phi \phi_{1} \alpha^{2} X_{V}\left(b^{2}\right)
\end{aligned}
$$

Note that

$$
X_{V}\left(\alpha^{2}\right)=2 V_{0 ; 0}, \quad X_{V}(\beta)=\left(V^{j} b_{i ; j}+b^{j} V_{j ; i}\right) y^{i} .
$$

Then (15) is equivalent to

$$
\left(\phi-\phi_{2} s\right) V_{0 ; 0}+\alpha \phi_{2}\left(V^{j} b_{i ; j}+b^{j} V_{j ; i}\right) y^{i}+\left(\phi_{1} X_{V}\left(b^{2}\right)-2 c \phi\right) \alpha^{2}=0 .
$$

In order to simplify the computation, at a fixed point $x \in M$, we make a coordinate change such that

$$
y^{1}=\frac{s}{\sqrt{b^{2}-s^{2}}} \bar{\alpha}, \quad \alpha=\frac{b}{\sqrt{b^{2}-s^{2}}} \bar{\alpha}, \quad \beta=\frac{b s}{\sqrt{b^{2}-s^{2}}} \bar{\alpha}, \bar{\alpha}=\sqrt{\sum_{a=2}^{n}\left(y^{a}\right)^{2}} .
$$

Then we have

$$
\begin{gathered}
V_{0 ; 0}=V_{1 ; 1} \frac{s^{2}}{b^{2}-s^{2}} \bar{\alpha}^{2}+\left(\bar{V}_{1 ; 0}+\bar{V}_{0 ; 1}\right) \frac{s}{\sqrt{b^{2}-s^{2}}} \bar{\alpha}+\bar{V}_{0 ; 0}, \\
\left(V^{j} b_{i ; j}+b^{j} V_{j ; i}\right) y^{i}=\left(V^{j} b_{1 ; j}+b^{j} V_{j ; 1}\right) \frac{s}{\sqrt{b^{2}-s^{2}}} \bar{\alpha}+\left(V^{j} \bar{b}_{0 ; j}+b^{j} \bar{V}_{j ; 0}\right),
\end{gathered}
$$

where

$$
\begin{gathered}
\bar{V}_{1 ; 0}+\bar{V}_{0 ; 1}=\sum_{a=2}^{n}\left(V_{1 ; a}+V_{a ; 1}\right) y^{a}, \quad \bar{V}_{0 ; 0}=\sum_{a, b=2}^{n} V_{a ; b} y^{a} y^{b} \\
V^{j} \bar{b}_{0 ; j}+b^{j} \bar{V}_{j ; 0}=\Sigma_{a=2}^{n}\left(V^{j} b_{a ; j}+b^{j} V_{j ; a}\right) y^{a} .
\end{gathered}
$$

Plugging (18) and (19) into (17), we have

$$
\begin{aligned}
& \left(\phi-\phi_{2} s\right)\left\{V_{1 ; 1} \frac{s^{2}}{b^{2}-s^{2}} \bar{\alpha}^{2}+\left(\bar{V}_{1 ; 0}+\bar{V}_{0 ; 1}\right) \frac{s}{\sqrt{b^{2}-s^{2}}} \bar{\alpha}+\bar{V}_{0 ; 0}\right\} \\
& +\phi_{2} \frac{b}{\sqrt{b^{2}-s^{2}}} \bar{\alpha}\left\{\left(V^{j} b_{1 ; j}+b^{j} V_{j ; 1}\right) \frac{s}{\sqrt{b^{2}-s^{2}}} \bar{\alpha}+\left(V^{j} \bar{b}_{0 ; j}+b^{j} \bar{V}_{j ; 0}\right)\right\} \\
& +\left\{\phi_{1} X_{V}\left(b^{2}\right)-2 c \phi\right\} \frac{b^{2}}{b^{2}-s^{2}} \bar{\alpha}^{2}=0 .
\end{aligned}
$$

Let

$$
\phi=a_{0}+a_{1} s+a_{2} s^{2}+o\left(s^{2}\right)
$$

with $a_{i}=a_{i}\left(b^{2}\right)$. Then

$$
\phi_{1}=a_{0}^{\prime}+a_{1}^{\prime} s+a_{2}^{\prime} s^{2}+o\left(s^{2}\right) .
$$

Firstly, let $s=0$ in (20), we have

$$
a_{0} \bar{V}_{0 ; 0}+a_{1}\left(V^{j} \bar{b}_{0 ; j}+b^{j} \bar{V}_{j ; 0}\right) \bar{\alpha}+\left\{a_{0}^{\prime} X_{V}\left(b^{2}\right)-2 c a_{0}\right\} \bar{\alpha}^{2}=0 .
$$


By the irrationality of $\bar{\alpha},(21)$ is equivalent to

$$
\begin{gathered}
a_{1}\left(V^{j} \bar{b}_{0 ; j}+b^{j} \bar{V}_{j ; 0}\right)=0, \\
a_{0} \bar{V}_{0 ; 0}+\left\{a_{0}^{\prime} X_{V}\left(b^{2}\right)-2 c a_{0}\right\} \bar{\alpha}^{2}=0 .
\end{gathered}
$$

Since $a_{1} \neq 0$ by assumption, then by (22) we have

$$
V^{j} \bar{b}_{0 ; j}+b^{j} \bar{V}_{j ; 0}=0
$$

which is equivalent to

$$
V^{j} b_{a ; j}+b^{j} V_{j ; a}=0
$$

By (23), we have

$$
V_{a ; b}+V_{b ; a}=-2\left\{\frac{a_{0}^{\prime}}{a_{0}} X_{V}\left(b^{2}\right)-2 c\right\} \delta_{a b}, \quad(2 \leq a, b \leq n)
$$

By the irrationality of $\bar{\alpha}$ again, from (20), we get

$$
\begin{gathered}
\left(\phi-\phi_{2} s\right)\left(\bar{V}_{1 ; 0}+\bar{V}_{0 ; 1}\right) \frac{s}{\sqrt{b^{2}-s^{2}}} \bar{\alpha}=0 . \\
\left(\phi-\phi_{2} s\right)\left\{V_{1 ; 1} \frac{s^{2}}{b^{2}-s^{2}} \bar{\alpha}^{2}+\bar{V}_{0 ; 0}\right\}+\phi_{2} \frac{b s}{b^{2}-s^{2}} \bar{\alpha}^{2}\left(V^{j} b_{1 ; j}+b^{j} V_{j ; 1}\right) \\
+\left\{\phi_{1} X_{V}\left(b^{2}\right)-2 c \phi\right\} \frac{b^{2}}{b^{2}-s^{2}} \bar{\alpha}^{2}=0 .
\end{gathered}
$$

From (26) we get

$$
\bar{V}_{1 ; 0}+\bar{V}_{0 ; 1}=0
$$

which is equivalent to

$$
V_{1 ; a}+V_{a ; 1}=0 .
$$

Solving (23) for $\bar{V}_{0 ; 0}$ and plugging it into (27), we have

$$
\begin{aligned}
& s^{2}\left(\phi-\phi_{2} s\right)\left\{V_{1 ; 1}+\left(\frac{a_{0}^{\prime}}{a_{0}} X_{V}\left(b^{2}\right)-2 c\right)\right\}-\left\{\frac{a_{0}^{\prime}}{a_{0}} X_{V}\left(b^{2}\right)-2 c\right\}\left(\phi-\phi_{2} s\right) b^{2} \\
& +\phi_{2} s b\left(V^{j} b_{1 ; j}+b^{j} V_{j ; 1}\right)+\phi_{1} b^{2} X_{V}\left(b^{2}\right)-2 c b^{2} \phi=0 .
\end{aligned}
$$

Expand $\phi\left(b^{2}, s\right)$ as Taylor series, that is $\phi=\Sigma_{k=0}^{\infty} a_{k} s^{k}$, where $a_{k}=\frac{1}{k !} \frac{\partial^{k} a_{0}}{\partial s^{k}}$. Plugging it into (29), and by the coefficient of $s$, we have

$$
b a_{1}\left(V^{j} b_{1 ; j}+b^{j} V_{j ; 1}\right)+b^{2} X_{V}\left(b^{2}\right) \frac{\partial a_{1}}{\partial\left(b^{2}\right)}-2 c b^{2} a_{1}=0 .
$$

Then

$$
V^{j} b_{1 ; j}+b^{j} V_{j ; 1}=-\left(\frac{a_{1}^{\prime}}{a_{1}} X_{V}\left(b^{2}\right)-2 c\right) b .
$$

Then by (24) and (31) we have

$$
V^{j} b_{i ; j}+b^{j} V_{j ; i}=-\left(\frac{a_{1}^{\prime}}{a_{1}} X_{V}\left(b^{2}\right)-2 c\right) b_{i} .
$$


Substituting (31) into (29), we have

$$
\begin{aligned}
& \left(\phi-\phi_{2} s\right) s^{2}\left\{V_{1 ; 1}+\left(\frac{a_{0}^{\prime}}{a_{0}} X_{V}\left(b^{2}\right)-2 c\right)\right\} \\
& -b^{2} X_{V}\left(b^{2}\right)\left\{\frac{a_{0}^{\prime}}{a_{0}}\left(\phi-\phi_{2} s\right)-\phi_{1}+\phi_{2} s \frac{a_{1}^{\prime}}{a_{1}}\right\}=0 .
\end{aligned}
$$

The coefficients of all powers of $s$ must vanish in (33). In particular, the coefficient of $s^{2}$ vanishes. We have

$$
V_{1 ; 1}+\frac{a_{0}^{\prime}}{a_{0}} X_{V}\left(b^{2}\right)-2 c b=-b^{2} X_{V}\left(b^{2}\right)\left[\frac{a_{0}^{\prime}}{a_{0}} \frac{a_{2}}{a_{0}}+\frac{a_{2}^{\prime}}{a_{0}}-2 \frac{a_{1}^{\prime}}{a_{1}} \frac{a_{2}}{a_{0}}\right] .
$$

By (25), (28), (34), we have

$$
\begin{aligned}
V_{i ; j}+ & V_{j ; i}=4 c a_{i j} \\
& -2 X_{V}\left(b^{2}\right)\left\{\frac{a_{0}^{\prime}}{a_{0}} a_{i j}+\left[\frac{a_{0}^{\prime}}{a_{0}} \frac{a_{2}}{a_{0}}+\frac{a_{2}^{\prime}}{a_{0}}-2 \frac{a_{1}^{\prime}}{a_{1}} \frac{a_{2}}{a_{0}}\right] b_{i} b_{j}\right\} .
\end{aligned}
$$

Plugging (34) into (33) yields

$$
b^{2} X_{V}\left(b^{2}\right)\left\{\left(\phi-s \phi_{2}\right)\left\{s^{2}\left[\frac{a_{0}^{\prime}}{a_{0}} \frac{a_{2}}{a_{0}}+\frac{a_{2}^{\prime}}{a_{0}}-2 \frac{a_{1}^{\prime}}{a_{1}} \frac{a_{2}}{a_{0}}\right]+\frac{a_{0}^{\prime}}{a_{0}}\right\}-\phi_{1}+s \phi_{2} \frac{a_{1}^{\prime}}{a_{1}}\right\}=0 .
$$

Suppose that $\phi=\phi\left(b^{2}, s\right)$ satisfies :

$$
\left(\phi-s \phi_{2}\right)\left\{s^{2}\left[\frac{a_{0}^{\prime}}{a_{0}} \frac{a_{2}}{a_{0}}+\frac{a_{2}^{\prime}}{a_{0}}-2 \frac{a_{1}^{\prime}}{a_{1}} \frac{a_{2}}{a_{0}}\right]+\frac{a_{0}^{\prime}}{a_{0}}\right\}-\phi_{1}+s \phi_{2} \frac{a_{1}^{\prime}}{a_{1}} \neq 0 .
$$

Then it follows from (36) that

$$
X_{V}\left(b^{2}\right)=0 .
$$

Then (33) is reduced to the following equation

$$
V_{1 ; 1}=2 c .
$$

By (25), (28), (38), we have

$$
V_{i ; j}+V_{j ; i}=4 c a_{i j} .
$$

In this case, (32) is reduced to

$$
V^{j} b_{i ; j}+b^{j} V_{j ; i}=2 c b_{i}
$$

Now we assume that $\phi=\phi\left(b^{2}, s\right)$ satisfies

$$
\left(\phi-s \phi_{2}\right)\left\{s^{2}\left[\frac{a_{0}^{\prime}}{a_{0}} \frac{a_{2}}{a_{0}}+\frac{a_{2}^{\prime}}{a_{0}}-2 \frac{a_{1}^{\prime}}{a_{1}} \frac{a_{2}}{a_{0}}\right]+\frac{a_{0}^{\prime}}{a_{0}}\right\}-\phi_{1}+s \phi_{2} \frac{a_{1}^{\prime}}{a_{1}}=0 .
$$

In this case, we are unable to show that $X_{V}\left(b^{2}\right)=0$ without additional condition on $\phi$.

Contracting (35) with $b^{i}$ and $b^{j}$ yields

$$
\begin{aligned}
V_{i ; j} b^{i} b^{j}= & 2 c b^{2} \\
& -b^{2} X_{V}\left(b^{2}\right)\left\{\frac{a_{0}^{\prime}}{a_{0}}+\left[\frac{a_{0}^{\prime}}{a_{0}} \frac{a_{2}}{a_{0}}+\frac{a_{2}^{\prime}}{a_{0}}-2 \frac{a_{1}^{\prime}}{a_{1}} \frac{a_{2}}{a_{0}}\right] b^{2}\right\} .
\end{aligned}
$$


Contracting (32) with $b^{i}$ and $b^{j}$ yields

$$
V_{i ; j} b^{i} b^{j}=2 c b^{2}-b^{2} X_{V}\left(b^{2}\right)\left\{\frac{1}{2 b^{2}}+\frac{a_{1}^{\prime}}{a_{1}}\right\} .
$$

Here, we used the fact that $X_{V}\left(b^{2}\right)=2 b_{i ; k} b^{i} V^{k}$. Then comparing (40) with (41) yields

$$
X_{V}\left(b^{2}\right)\left\{\frac{1}{2 b^{2}}+\frac{a_{1}^{\prime}}{a_{1}}-\frac{a_{0}^{\prime}}{a_{0}}-\left[\frac{a_{0}^{\prime}}{a_{0}} \frac{a_{2}}{a_{0}}+\frac{a_{2}^{\prime}}{a_{0}}-2 \frac{a_{1}^{\prime}}{a_{1}} \frac{a_{2}}{a_{0}}\right] b^{2}\right\}=0,
$$

Thus if

$$
\frac{1}{2 b^{2}}+\frac{a_{1}^{\prime}}{a_{1}}-\frac{a_{0}^{\prime}}{a_{0}}+\left\{\frac{a_{0}^{\prime}}{a_{0}}\left[2 \frac{a_{1}^{\prime}}{a_{1}}-\frac{a_{0}^{\prime}}{a_{0}}\right]-\frac{a_{2}}{a_{0}}\right\} b^{2} \neq 0,
$$

then $X_{V}\left(b^{2}\right)=0$.

Q.E.D.

\section{Proofs of Theorems 1.3 and 1.4}

. In this section, we are going to prove Theorems 1.3 and 1.4 , that is, we shall solve conformal vector fields from the PDEs in the theorems when $F$ is a spherically symmetric metric.

Proof of Theorem 1.3: Let $F=|y| \phi\left(|x|^{2}, \frac{\langle x, y\rangle}{|y|}\right)$ be a spherically symmetric metric satisfying the conditions of Theorem 1.2. If $V$ is a conformal vector field of $F$, then $X_{V}\left(b^{2}\right)=2 \sum_{j=1}^{m} V^{j} x^{j}=0$. By the second equation of (4), one has $0=\frac{\partial\left(\sum_{j=1}^{m} V^{j} x^{j}\right)}{\partial x^{i}}=2 c x^{i}$. Hence $c=0$ and the first equation of $(4)$ is reduced to

$$
\frac{\partial V^{i}}{\partial x^{j}}+\frac{\partial V^{j}}{\partial x^{i}}=0
$$

The solution of $(44)$ is $([1])$

$$
V^{i}=q_{k}^{i} x^{k}+\eta^{i}
$$

where $\left(q_{k}^{i}\right)$ is an anti-symmetric matrix and $\eta^{i}$ are constants. Note that $0=$ $X_{V}\left(b^{2}\right)=2 \sum_{j=1}^{m} V^{j} x^{j}=2\langle\eta, x\rangle$, hence $\eta=0$ and then $V^{i}=q_{k}^{i} x^{k}$. This completes the proof.

Proof of Theorem 1.4: For the functions $a_{0}, a_{1}$ and $a_{2}$ in (10), we have

$$
\frac{a_{0}^{\prime}}{a_{0}}=\frac{2 \delta-1}{2|x|^{2}}, \quad \frac{a_{1}^{\prime}}{a_{1}}=\frac{2 \delta-2}{2|x|^{2}}, \quad \frac{a_{2}^{\prime}}{a_{2}}=\frac{2 \delta-3}{2|x|^{2}} .
$$

Thus

$$
\frac{1}{2|x|^{2}}+\frac{a_{1}^{\prime}}{a_{1}}-\frac{a_{0}^{\prime}}{a_{0}}=0, \quad \frac{a_{2}}{a_{0}}\left[2 \frac{a_{1}^{\prime}}{a_{1}}-\frac{a_{0}^{\prime}}{a_{0}}\right]-\frac{a_{2}^{\prime}}{a_{0}}=0 .
$$

Since $a_{i j}=\delta_{i j}, b_{i}=x^{i}$ and $h:=\frac{1}{2} X_{V}\left(|x|^{2}\right)=\sum_{i=1}^{n} V^{i} x^{i},(6)$ and $(7)$ are reduced to

$$
\begin{aligned}
& \frac{\partial V^{i}}{\partial x^{j}}+\frac{\partial V^{j}}{\partial x^{i}}=4 \bar{c} \delta_{i j}, \\
& \frac{\partial h}{\partial x^{i}}+2 h \frac{a_{1}^{\prime}}{a_{1}} x^{i}=2 c x^{i},
\end{aligned}
$$


where $\bar{c}=c-\frac{a_{0}^{\prime}}{a_{0}} h$. The general solution to (46) is given by ([10]):

$$
\begin{gathered}
V^{i}=-2(\lambda+<d, x>) x^{i}+|x|^{2} d^{i}+q_{r}^{i} x^{r}+\eta^{i}, \\
\bar{c}=-\lambda-<d, x>,
\end{gathered}
$$

where $\lambda$ is a constant number, $d, \eta$ are constant vectors and $\left(q_{r}^{i}\right)$ is constant antisymmetric matrix. In this case, $h$ and $c$ given by the following equations

$$
\begin{gathered}
h=-2 \lambda|x|^{2}-|x|^{2}<d, x>+<\eta, x>, \\
c=-\lambda-<d, x>+\frac{a_{0}^{\prime}}{a_{0}} h .
\end{gathered}
$$

Plugging (50) and (51) into (47) yields:

$$
<d, x>x^{i}-\frac{<\eta, x>}{|x|^{2}} x^{i}=|x|^{2} d^{i}-\eta^{i} .
$$

from which we can easily see that $d=\eta=0$. Then we have

$$
V^{i}=-2 \lambda x^{i}+q_{k}^{i} x^{k} .
$$

and

$$
c=-\lambda-2 \lambda \frac{a_{0}^{\prime}}{a_{0}}|x|^{2}=-2 \delta \lambda
$$

Q.E.D.

\section{References}

[1] D. Bao, C. Robles and Z. Shen, Zermolo navigation on Riemann manifolds, J. Diff. Geom. 66(2004), 391-449.

[2] L. Huang and X. Mo, On spherically symmetric Finsler metrics of scalar curvature, J. Geom. Phys.,62(2012), 2279-2287.

[3] L.Kang, On conformal fields of $(\alpha, \beta)$-space, preprint, 2011.

[4] X. Mo and L. Zhou, The curvature of spherically symmetric Finsler metrics in $R^{n}$, preprint

[5] X. Mo, L. Zhou and H. Zhu, On spherically symmetric Finsler metrics of constant curvature, preprint, November 2013.

[6] E.S. Sevim, Z. Shen and S. Ulgen, Spherically symmetric Finsler metrics with constant Ricci and flag curvature, preprint, October 2014.

[7] Z. Shen and Q. Xia, On conformal vector fields on Randers manifolds, Sci. in China, 55(9)(2012), 1869-1882.

[8] Z. Shen and Q. Xia, A class of Randers metrics of scalar flag curvature, Internat. J. Math. 24(2013), 1350055, 9. 
[9] Z. Shen and Q. Xia, Conformal vector fields on a locally projectively flat Randers manifold, Publ. Math. Debreceen, 84(3-4), 2014.

[10] Z. Shen and H. Xing, On Randers metrics of isotropic S-curvature, Acta. Math. Sin., English Series, 24(2008), 789-796.

[11] C. Yu and H. Zhu, On a new class of Finsler metrics, Diff. Geom. Appl. 29(2011), 244-254.

[12] L. Zhou, Projective spherically symmetric Finsler metrics with constant flag curvature in $R^{n}$, Geom. Dedicata, 158(2012), 353-364.

Department of Mathematical Sciences, Indiana University Purdue University Indianapolis

402 N Blackford Street, Indianapolis, IN 46202, USA

E-mail: zshen@math.iupui.edu, mingyuan@iupui.edu 